\title{
The rotating square illusion
}

\author{
ENNIO MINGOLLA and JAMES TODD \\ University of Connecticut, Storrs, Connecticut 06268
}

\begin{abstract}
Twenty subjects viewed displays of square and trapezoidal luminous dot figures rotating about a vertical axis in both polar and parallel projections. The frequency of reported oscillation of the rotating figures was as high for the squares as for the trapezoids, provided that the squares contained static slant information in the form of internal texture gradients. Previously reported oscillation effects using shapes other than trapezoids have generally been unreliable, with a subject as likely to see rotation as oscillation for a given cycle, and then only in parallel projection. As a consequence, linear perspective information in trapezoidal shape has been taken to be the indispensable element for obtaining a strong oscillation effect. The authors argue that in the construction of the rotating object, a required condition for reliably producing the effect is a violation of some optical constraint regarding slant information, not necessarily one involving linear perspective.
\end{abstract}

The name "trapezoidal window illusion" overstates the importance of trapezoidal shape in promoting the experienced oscillation of rotating figures. The strong oscillation effect is a function of slant information, of which linear perspective contained in trapezoidal contours is but a single example. In the classical form of the illusion (Ames, 1951), subjects viewing trapezoidal window displays typically see the actual rotary motion of the window as oscillatory motion, with the longer vertical edge always closer. Ames' original explanation of the illusion is based on the premise that an observer's familiarity with trapezoidal shapes in the visual field creates an expectation that such shapes correspond to rectangular physical objects at a slant to the line of sight. The dimensions of the Ames window and the viewing conditions are such that the optical projection of the window at the observer's eye is always trapezoidal, with the physically longer vertical edge always casting the longer optical projection. This creates the impression that the physically longer edge is closer to the observer, even when it is in fact farther away.

Since the publication of Ames' study, many variations of shape, interior contours, and shading have been employed in displays designed to elicit the oscillation experience (Ames, 1951; Braunstein, 1971; Freeman \& Pasnak, 1968; Pastore, 1952; Power, 1967). As the name "trapezoidal window" implies, however, most of the explanations of the effect have concentrated on the shape of the rotating object, construing the orientations of the top and bottom edges of the trapezoid as embodying the depth or slant cue of linear perspective. The present experiment demonstrates that the oscillation ef fect obtained with the Ames window is not a function of the trapezoidal shape of the window as such, but rather can be obtained through creation of various optical displays that violate environmental constraints regarding slant information.

Despite their theoretical differences, most past researchers have agreed that misjudgment of the slant of the window to the line of sight is a central component of the oscillation effect (Ames, 1951; Graham, 1963; Pastore, 1952; Power \& Day, 1973). Some researchers have calculated the particular values of slant that are suggested by linear perspective cues throughout the rotation cycle of the window (Freeman \& Pasnak, 1968). Others have paid particular attention to ambiguity in the cue of motion parallax, which is resolved by linear perspective information in the window's shape (Braden, 1978; Day \& Power, 1965; Graham, 1963; Graham \& Gillam, 1970), although Hershberger (1967) has warned that, for trapezoidal displays, the ambiguity exists only in parallel projection. To repeat, however, linear perspective is not a privileged source of information for surface slant. Several kinds of information for slant, most involving optical texture, have been identified since the publication in 1950 of Gibson's seminal work, The Perception of the Visual World.

Identified sources of slant information in static displays include: texture density gradients (Flock, 1964a; Gibson, 1950b; Gruber \& Clark, 1956), texture size and shape gradients (Flock \& Moscatelli, 1964; Phillips, 1970), compression of texture elements parallel to the axis of rotation and convergence perpendicular to the axis (Attneave \& Olson, 1966; Gillam, 1968), contour convergence or linear perspective (Clark, Smith, \& Rabe, 1956; Freeman, 1966), form ratio (Braunstein \& Payne, 1968), and binocular disparity (Gillam, 1968; Ogle, 1950). Dynamic transformation of ten increases the accuracy of slant judgments compared with those of static views. Identified sources of dynamic information for slant include: 
rotation to a slant (Gibson \& Gibson, 1957), motion parallax through translation of a surface in a direction orthogonal to its slant (Flock, 1962, 1964b), and changes in form ratio (Braunstein \& Payne, 1969).

All of the studies just cited investigated a single source of information or placed two sources in competition to determine which was perceptually more salient or which yielded more accurate judgments. Methodological concerns seemed to require isolation of sources of information, thereby fostering debate concerning the primacy of various sources, such as the exchange between Flock $(1964 a, 1965)$ and Freeman $(1965,1966 \mathrm{~b})$. Flock considered contour gradients containing linear perspective to be special cases of texture gradients, while Freeman considered texture gradients to be special cases of contour gradients.

Braunstein (1976) observes that both Flock (1964a, $1965)$ and Freeman $(1965,1966 \mathrm{~b})$ were in a sense correct and emphasizes that the same mathematical information for slant is available from each source, although he warns that the perceptual salience of different sources varies situationally. Braunstein's point can be expanded to include all sources of information about surface slant. For a naturally occurring surface in any orientation, sources of visual information veridically and redundantly specify the same value for slant to the line of sight. This claim is central to the motivation of the present experiment and bears elaboration in some detail.

Implicit in the phrase "naturally occurring surface" are certain suppositions restricting the physical characteristics of that surface and consequently the nature of any optical display involving that surface. Formulations of such restrictions are called "optical constraints" and vary from the probabilistic to the virtually absolute. Ames' (1951) view involves the supposition that straight contoured quadrilateral shapes in our environment tend to be rectangles, so trapezoidal forms in an optical display probably represent rectangular surfaces at a slant. Kaplan's (1969) work involves a considerably stronger premise that physical surfaces can neither be created from nothing nor disappear without a trace. Consequently, the appearance or disappearance of texture elements in an optical display provides conclusive grounds for perceiving an edge of one surface occluding another surface. Kinetic random dot displays exploiting only this constraint provide a vivid experience of a surface edge even in the absence of any contour of lightness contrast such as might be presumed necessary to see an edge.

Optical texture is formed through the adjacent ordering of discontinuities of lightness values in a display. Constraints regarding optical texture express the necessary relations between such optical discontinuities and corresponding orderings of reflectance values both across and within surfaces due to variations in the physical and chemical characteristics of the surfaces. The essential environmental constraint guaranteeing that optical texture information is veridical is that the physical or chemical texture of surfaces be either regular or stochastically regular (Gibson, 1979). Thus, equal numbers of similar optical texture elements correspond to equal surface areas, despite differences in the sizes of optical projections of those elements. Several grains of texture may be nested in a single surface. A simple case of nesting is a brick sidewalk, in which the bricks form a coarse unit of texture with regular patterning, while within each brick, surface irregularities provide a finer, stochastically regular texture.

Once optical constraints concerning surfaces at a slant are identified, displays in which optical information indicates a nonveridical slant of a physical object are relatively easy to contrive. In the classic rotating trapezoid illusion, the constraint regarding the probable shape of quadrilaterals is violated by producing a trapezoidal object, whose converging contours mimic the effect of parallel contours at a slant to the line of sight. In order to maximize the effect of outer contour, researchers generally attempt to eliminate fine surface texture along the window segments by using special display apparatus, such as shadow casters ((Braden, 1978; Graham \& Gillam, 1970 ) or luminous wire displays (Murch, 1970; Zegers \& Murray, Note 1). Even studies involving nonluminous physical objects have often combined highly reflective, homogeneous construction materials and illumination in such a manner as to minimize the perceptual salience of surface texture along figural segments (Ames, 1951; Day \& Power, 1965; Freeman \& Pasnak, 1968). When an Ames window is filled with panes, however, the panes themselves form coarse texture units, which violate the constraint regarding distribution of similar texture elements on a surface; that is, an equal number of elements do not cover equal areas of the surface. Little has been made of this constraint violation, although Zegers (1965) has noted that the presence or absence of panes does not affect the number of reported oscillations.

Given the affinity already noted between perspective and slant information, and given researchers' implicit affirmation that textural information is important enough to be eliminated, the lack of a systematic investigation of texture constraints and oscillation effects is somewhat surprising. The present experiment does not seek to refute claims regarding manipulations of linear perspective and the Ames effect but to demonstrate that the same effect can be obtained through similar manipulations of other kinds of optical information.

When using trapezoidal figures, before any measurements are made concerning linear perspective, motion parallax, or any other optical information, an optical constraint regarding linear perspective must be violated in order to produce the figure to be rotated. If such a violation is a necessary con- 
dition for producing a strong oscillation effect, it should be possible to consistently evoke the effect in displays lacking a trapezoidal contour, simply by manipulating some other source of static slant information besides linear perspective.

While oscillation effects with rectangular-shaped displays have been reported previously, they have occurred only half the time for a given display in which visual information was ambiguous with regard to slant and direction of motion (Braden, 1978; Braunstein \& Payne, 1968; Cahill, 1969). In other words, the proportion of oscillation judgments for a display was at the chance level, given the choice of seeing a cycle as rotation or as oscillation. The present experiment demonstrates that oscillation judgments can be reliably elicited using displays having internal textural information but no linear perspective or trapezoidal contours.

\section{METHOD}

\section{Subjects}

Of the 20 naive subjects, 13 were members of an introductory psychology course who participated in order to fulfill a course requirement, while the remaining 7 were graduate student volunteers. All had normal or fully corrected vision.'

\section{Stimuli}

Computer-generated displays of luminous dots moved on a cathode ray tube (CRT) in such a manner as to simulate rotation of square or trapezoidal shapes about a vertical axis. Trajectories of the eight dot configurations depicted in Figure 1 were computed in both parallel and polar projections.

Each dot configuration in Figure 1 denotes the frame occurring whenever a virtual display object is in the plane of the CRT screen and therefore perpendicular to the line of sight. The positions of the dots in each display specify in an abstract manner values for linear perspective and for gradients of vertical optical convergence and horizontal optical compression as follows: Displays $C, D, G$, and $H$ show linear perspective along their outer contour, with the rightmost vertical edge being half the length of the leftmost. Displays $B, D, F$, and $H$ show a corresponding gradient value for convergence of dots within vertical contours, with dots of the rightmost edge being twice as dense as those of the leftmost. Displays $E, F, G$, and $H$ show the corresponding gradient of compression of vertical contours from left to right. The gradients specify identical values of surface slant. Display $\mathbf{H}$ is the only one depicting a rectangular surface at a slant through all three sources of information, although it must be remembered that this display

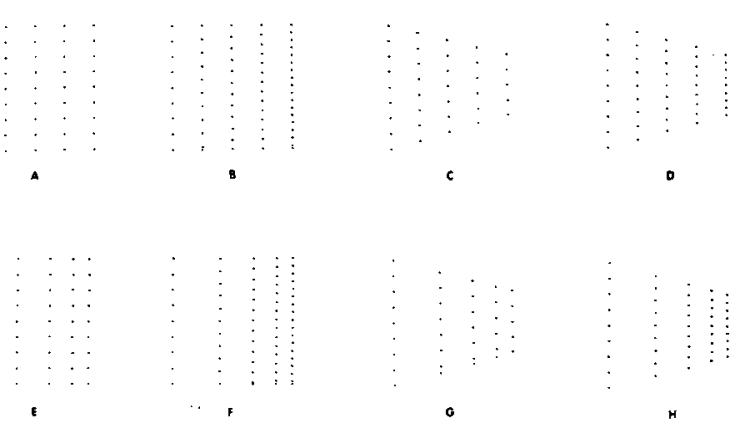

Figure 1. Frames of display objects when perpendicular to the line of sight. represents the dot configuration occurring when the rotating object is perpendicular to the line of sight. Display $A$ is thus the only one that unequivocally depicts a surface perpendicular to the line of sight when actually in that orientation.

The eight displays depicted in Figure 1 exhaust all possible combinations of corresponding positive or zero values for the three static sources of slant information in the displays. Parallel projections of dot positions through rotation cycles were calculated for half the trials. The other half of the trials employed a polar projection calculated to correspond to the subjects' actual viewing distance, approximately $60 \mathrm{~cm}$. Following Braunstein (1976), the degree of polar projection can be expressed as the ratio of two distances from an observation point. When perpendicular to the line of sight, the distance of the farthest edge divided by the distance to the closest edge was 1.18 for all displays. The vertical axis of rotation for all displays except $A$ passed through the centroid defined by one or more of the optical gradients, the centroid being, in each case, two-thirds the horizontal distance from the left edge of the display object. Display A was rotated about its center. The displays were approximately $10 \mathrm{~cm}$ wide when perpendicular to the line of sight. Each trial consisted of four continuous rotations of a given object in one of the projection conditions and took $22 \mathrm{sec}$. Four seconds elapsed between trials in a block.

\section{Apparatus}

Displays were presented on a Tektronix 611 CRT refreshed every $5 \mathrm{msec}$ by a Nova minicomputer. The displays were viewed binocularly at a distance of approximately $2 \mathrm{ft}$ in a darkened room. Head movements were not restricted.

\section{Procedure}

Before each subject saw any of the computer-generated displays, the experimenter manipulated a book to illustrate the difference between continuous rotation and oscillation about a vertical axis. Subjects were instructed to press a response key each time a display object appeared to change direction. The experimenter then left the room, and two blocks of 16 trials in randomized order were shown to the subject. There was a 30 -sec pause between blocks, and the entire experiment was completed in 20 min.

\section{RESULTS}

Each subject viewed each display twice in both projection conditions, and the reported oscillations for each pair of four rotation cycle trials were summed for each subject. Table 1 displays the mean reported oscillations for the eight rotation cycles for each condition. As predicted, Display A yielded fewer oscillation responses than any other in parallel projection; only $\mathrm{E}$ yielded fewer oscillation responses in polar projection. (See Figure 1.)

Two planned comparisons for each projection condition corroborate the main thesis of the present paper. The contention that the oscillation effect is a general consequence of violation of optical constraints regarding slant information is supported by comparing the mean for Display A against the weighted mean for Displays $B$ through $G$. The contention that trapezoidal shape is not a privileged condition for the oscillation effect is supported by comparing the weighted mean for Displays $C, D, G$, and $H$ against that for Displays B, E, and F. The former group contains all the trapezoidal shapes in the experiment, while the latter contains all the square shapes employing at least one of the two internal gradients. 
Table 1

Mean Reported Oscillations for Two Presentations of Each Display

\begin{tabular}{lrrrrrrrrr}
\hline \multicolumn{8}{c}{ Display } \\
\cline { 2 - 9 } & A & B & C & D & E & F & G & H \\
\hline \multicolumn{7}{c}{ Parallel Projection } \\
Mean & 8.95 & 13.15 & 13.75 & 11.85 & 10.95 & 13.60 & 14.25 & 11.00 \\
SD & 4.82 & 3.14 & 2.59 & 3.61 & 3.31 & 2.52 & 2.07 & 4.68 \\
& \multicolumn{8}{c}{ Polar Projection } \\
Mean & .60 & 3.15 & 4.50 & .70 & .25 & 4.20 & 4.85 & 1.05 \\
SD & .80 & 4.15 & 5.10 & .54 & .84 & 4.73 & 4.51 & 1.99 \\
\hline
\end{tabular}

Any contribution from trapezoidal shape exceeding or supplementing compression and convergence ought to be manifest as an effect in the weighted comparison.

The results of the planned comparisons are as follows. The weighted means for Displays B through G are $\mathbf{1 2 . 6 5}$ for parallel projection and 2.67 for 1.18 polar projection; corresponding means for Display $\mathbf{A}$ are 8.95 and .60 . This comparison is highly significant in both projection conditions, with $[\mathrm{F}(1,133)=25.57$, $p<.001]$ for parallel projection and $[F(1,133)=9.41$, $\mathrm{p}<.01$ ] for polar. In fact, for parallel projection, the difference between the mean for Display $A$ and that for the other seven accounts for over half of all the between-displays variance. In contrast, the second comparison yields no effect in either condition. Weighted means for square figures with internal gradients are 12.57 and 2.53 for parallel and polar projection, respectively; corresponding weighted means for trapezoidal figures are 12.71 and 2.78. Such differences hardly bear significance testing, but, to underscore the lack of even a tendency for an effect, $F(1,133)=.08$ for parallel projection and $F(1,133)=.25$ for polar. It appears that, at least for dot displays employing vivid texture information, the oscillation effect cannot be noticeably improved by the addition of trapezoidal outer contours. ${ }^{2}$

\section{DISCUSSION}

Previously reported oscillation effects for rectangular displays have occurred at chance levels for displays showing no static slant information (Braden, 1978; Braunstein \& Payne, 1968; Cahill, 1969). Display $A$ is the closest analog of such displays used in the present experiment. (See Figure 1.) Consistent with past studies, Display A showed a low frequency of oscillation judgments. In fact, after completing the experiment, several subjects reported difficulty in seeing a certain display, determined by description to be $A$ in parallel projection, as even rotating. They experienced "accordion-like" motion in the plane instead, consistent with reports in classic studies using displays with similar harmonic motion trajectories in a single dimension (Johansson, 1964; Wallach \& O'Connell, 1953). In the present experiment, how- ever, oscillation judgments for displays with square outlines were consistently elicited using parallel projection, provided figures containing compression or convergence gradients were used. Even the perceptually salient 1.18 polar projection was insufficient to completely damp the oscillation effect for four of the displays including two with square outlines. This last point requires some elaboration.

Braunstein (1976) describes how surface slant may be optically specified during a rotation by changes in form ratio, which he defines as the relative horizontal and vertical displacement of projected points. While changes in form ratio specify degree of surface slant in either projection, they can specify only direction of slant in polar projection. Thus, with the vertical rotations employed in trapezoidal window displays, changes in form ratio in parallel projection do not specify whether the left or right edge of the object is at any moment closer, accounting for the dramatic difference in oscillation judgments across the two projections.

The damping of oscillation judgments across degrees of polarity of projection was first systematically investigated by Braunstein and Payne (1968). Their subjects' task was to position a switch to indicate whether a given display object was at that moment rotating in a clockwise or counterclockwise direction. Subjects' responses indicated whether they saw the direction of rotation correctly or incorrectly, or whether they were experiencing the oscillation effect. Rectangular displays were seen to oscillate about half the time in parallel projection, and accuracy of rotation judgments for such displays increased markedly as polar projection values were increased by steps from 1.0 to 2.0. On the basis of the present experiment, one would expect that, using displays with rectangular outlines but sufficiently salient internal texture gradients, oscillation effects could be produced at some polarity values at which Braunstein and Payne (1968) report consistently accurate rotation judgments. ${ }^{3}$

In agreement with several findings in the literature, the present experiment demonstrates that no single source of visual information is uniquely responsible for the oscillation effect. Previous researchers have demonstrated that the oscillation effect can be enhanced by a wide variety of display properties that normally indicate an object's slant or direction of motion. These properties include shading (Cross \& Cross, 1969), interposition of individual display elements (Braunstein \& Stern, 1980), and linear perspective as revealed by the relative lengths of vertical contours (Braden, 1978) or by the relative angles formed at different vertices (Braunstein, 1971).

No previously proposed explanation of the rotating trapezoid illusion can account for all of these effects. Most proposed explanations have argued that a specific source of information is most responsible for the experienced orientation of an object or its apparent 
direction of motion. Some investigators, such as Graham (1963), have argued for the primacy of static sources of information, such as linear perspective or relative size. Others, including Braunstein (1972), have claimed that dynamic factors, such as changes in projected contour angles, are most influential. The present experiment is based on an alternate premise that the presence of some source of false information about the slant of a surface or its direction of motion is a necessary condition for producing an oscillation effect, provided that the observer is sensitive to such information and that competing sources of veridical information are minimized. Unfortunately, while all studies reporting an oscillation effect describe certain sufficient conditions for producing the effect, a general account of sufficient conditions on constraint violations and the presence of competing veridical information is still lacking.

Central to any investigation of information in optical displays, such as rotating trapezoids, is the experimenter's view of the relations that patterns in an optical display bear to objects and events in the natural environment. In the present study, such relations are characterized by constraints, which are taken to provide a basis for optical information. The projected shapes of objects on a display screen could not be informative, even in principle, about the shapes of the three-dimensional objects without ecological constraints, whether absolute or probabilistic, on the shapes of those objects. Similarly, information involving texture patterns of a visual display depends on constraints on the distribution of physical or chemical texture on objects. The patterns of shading in a visual display would be uninformative without constraints regarding the reflection of light from physical surfaces. Finally, motion patterns on a display screen are only informative because of constraints on the motion of objects in a natural environment. Under ordinary circumstances, the sources of visual information just named are completely redundant (Braunstein \& Payne, 1969). For example, when approaching a hill while driving, motion gradients, texture gradients, luminance gradients, and linear perspecitve all veridically specify the same value for the slant of the hill. In the laboratory, however, an observer often encounters artificially constructed displays, such as a rotating trapezoidal window, that violate certain constraints governing objects and events in natural settings. Such displays can be useful tools for studying an observer's sensitivity to optical information, provided the researcher is mindful that constraint violations may elicit observer reactions that are not representative of normal perception.

\section{REFERENCE NOTE}

1. Zegers, R. T., \& Murray, P. Perception of distortion. $\langle 1\rangle A n$ experimental approach to illusion (Navtradevcen 506-1). Port
Washington, N.Y: U.S. Naval Training Device Center, October 1962.

\section{REFERENCES}

Ames, A. Visual perception and the rotating trapezoidal window. Psychological Monographs, 1951, 67(7, Whole No. 324).

Attneave, F., \& Olson, R. K. Inferences about visual mechanisms from monocular depth effects. Psychonomic Science, $1966,4,133-134$.

Braden, J. H. The effect of varying linear perspective, movement parallax, and speed of rotation on perceived oscillation of twodimensional trapezoidal stimuli. Perception \& Psychophysics, $1978,23,51-57$.

Braunstein, M. L. Perception of rotation in figures with rectangular and trapezoidal features. Journal of Experimental Psychology, 1971, 91, 25-29.

Braunstein, M. L. Perception of rotation in depth: A process model. Journal of Experimental Psychology, 1972, 79, 510-524.

Braunstein, M. L. Depth perception through motion. New York: Academic Press, 1976.

Braunstein, M. L., \& Payne, J. W. Perspective and the rotating trapezoid. Journal of the Optical Society of America, 1968, 58, 399-403.

Braunstein, M. L., \& PAyne, J. W. Perspective and form ratio as determinants of relative slant judgments. Journal of Experimental Psychology, 1969, 81, 584-590.

Braunstein, M. L., \& Stern, K. R. Static and dynamic factors in the perception of rotary motion. Perception \& Psychophysics, $1980,27,313-320$.

CAHILL, M. Accuracy of position judgments of stationary targets yielded by three types of perceiver of the Ames trapezoidal illusion. Proceedings of the 77th Annual Convention of the A.P.A., 1969, 29-30.

Clark, W. C., Smith, A. H., \& RABe, A. The interaction of surface texture, outline gradient, and ground in the perception of slant. Canadian Journal of Psychology, 1956, 10, 1-8.

Cross, J., \& Cross, J. The misperception of rotary motion. Perception \& Psychophysics, 1969, 5, 94-96.

DAY, R. H., \& Powe R, R. P. Apparent reversal (oscillation) of rotary motion in depth: An investigation and a general theory. Psychological Review, 1965, 72, 117-127.

FLock, H. R. The monocular perception of surface slant (Doctoral dissertation, Cornell University, 1962). Ann Arbor: University Microfilms, 1962, No. 62-2514.

Flock, H. R. A possible optical basis for monocular slant perception. Psychological Review, 1964, 71, 380-391. (a)

FLock, H. R. Some conditions sufficient for accurate monocular perceptions of moving surface slants. Journal of Experimental Psychology, 1964, 67, 560-572. (b)

FLOCK, H. R. Optical texture and linear perspective as stimuli for slant perception. Psychological Review, 1965, 72, 505-514.

Flock, H. R., \& Moscatelli, A. Variables of surface texture and accuracy of space perceptions. Perceptual and Motor Skills, $1964,19,327-334$.

Freeman, R. B., Jr. Ecological optics and visual slant. Psychological Review, 1965, 72, 501-504.

Freeman, R. B., JR. Effect of size on visual slant. Journal of Experimental Psychology, 1966, 71, 96-103. (a)

Freeman, R. B., JR. Optical texture versus retinal perspective: A reply to Flock. Psychological Review, 1966, 73, 365-371. (b)

Freeman, R. B., \& Pasnak, R. Perspective determinants of the rotating trapezoid illusion. Journal of Experimental Psychology, 1968, 76, 94-101.

GiBson, J. J. The perception of the visual world. Boston: Houghton Mifflin, 1950. (a)

Gibson, J. J. The perception of visual surfaces. American Journal of Psychology, 1950, 63, 367-384. (b)

GIBSON, J. J. The ecological approach to visual perception. Boston: Houghton Mifflin, 1979. 
Gibson, J. J., \& Gibson, E. J. Continuous perspective transformations and the perception of rigid motion. Journal of Experimental Psychology, 1957, 54, 129-138.

Gillam, B. J. Perception of slant when perspective and stereopsis conflict: Experiments with aniseikonic lenses. Journal of Experimental Psychology, 1968, 78, 299-305.

GrahaM, C. H. On some aspects of real and apparent visual movement. Journal of the Optical Society of America, 1963, 53, 1019-1025.

Graham, C. H., \& Gillam, B. Occurrence of theoretically correct responses during rotation of the Ames window. Perception \& Psychophysics, 1970, 8, 257-260.

Gruber, H. E., \& Clark, W. C. Perception of slanted surfaces. Perceptual and Motor Skills, 1956, 6, 97-106.

Hershberger, W. A. Comments on "Apparent reversal (oscillation) of rotary motion in depth." Psychological Review, 1967, 74, 235-238.

Joh ANSson, G. Perception of motion and changing form. Scandinavian Journal of Psychology, 1964, 5, 181-208.

KAPLAN, G. Kinetic disruption of optical texture: Perception of depth at an edge. Perception \& Psychophysics, 1969, 6, 193-198.

Murch, G. M. Perception of rotary movement. Journal of Experimental Psychology, 1970, 86, 83-85.

OGLE, K. N. Researches in binocular vision. New York: Hafner, 1950.

Pastore, N. Some remarks on the Ames oscillatory effect. Psychological Review, 1952, 59, 319-323.

Phillips, R. J. Stationary visual texture and the estimation of slant angle. Quarterly Journal of Experimental Psychology, 1970, 22, 389-397.

Powe R, R. P. Stimulus properties which reduce apparent reversal of rotating rectangular shapes. Journal of Experimental Psychology, 1967, 73, 595-599.

Power, R. P., \& DAY, R. H. Constancy and illusion of apparent direction of rotary motion in depth: Tests of a theory. Perception \& Psychophysics, 1973, 13, 217-223.

Wallach, H., \& O'Connell, D. N. The kinetic depth effect. Journal of Experimental Psychology, 1953, 45, 205-217.

ZEGERS, R. T. The reversal illusion of the Ames trapezoid. Translations of the New York Academy of Sciences, 1965, 26, 377-400.

\section{NOTES}

1. Two of an original 22 subjects emerged from the experiment complaining that they were confused and were not sure they had seen what they were "supposed" to see. Their oscillation reports were much fewer than those of any other subjects and were scattered, seemingly at random, among displays and projections. These two subjects were dropped from the analysis.

2. To deny the efficacy of trapezoidal shape in Comparison 2 is in effect to argue for the null hypothesis. Here, however, there is no evidence that a small effect might go undetected due to power limitations. A skeptic might object that, at least for parallel projection, the data show a ceiling effect, making the task of increasing the oscillation effect through trapezoidal contours an unfair one. The objection, however, is tantamount to admitting that the rectangular displays with internal texture gradi. ents produce as powerful an effect as one can register with what is, after all, a natural response measure. Moreover, trapezoidal shape does not increase reported oscillations in the polar projection, in which plenty of room for increases exists.

3. Braunstein and Payne (1968) employed a display composed of intersecting horizontal and vertical lines, which they called a "horizontal perspective trapezoid," and which was directly analogous to Display $E$ in the present study. In the parallel projection condition, their subjects reported the oscillation effect $65 \%$ of the time. This lower proportion of oscillation judgments may be in part a function of differing response measures or use of dark line displays instead of luminous dots. It seems likely, however, that the main reason is the lower compression gradient value in their display; that is, the rate of left to right compression of vertical contours is greater in the present experiment. In any event, Braunstein and Payne (1968) did not pursue the question of internal texture gradients. Braunstein and Stern (1980) again used a figure analogous to Display $E$ of the present experiment, this time evoking a negligible oscillation effect. As before, though, the gradient value was lower than the one used in the present study.

(Received for publication June 30, 1980; accepted March 31, 1981.) 